\title{
Radiation therapy for primary and metastatic tumors of the liver
}

\author{
Chiao-Ling Tsai $\cdot$ Hans T. Chung • William Chu • \\ Jason Chia-Hsien Cheng
}

Received: 14 May 2012 / Accepted: 22 May 2012 / Published online: 6 June 2012

(C) Springer-Verlag 2012

\begin{abstract}
Background Hepatic tumors can be either primary disease (i.e., hepatocellular carcinoma) or a site of metastases from other solid malignancies. The primary therapy for all liver tumors is resection, but only a minority of patients present with resectable disease. Various nonsurgical ablative techniques have been investigated to cure or palliate unresectable hepatic tumors by improving locoregional control.

Purpose The objective of this article is to review the radiotherapy options in managing primary or metastatic liver cancer. Design and methods Two emerging radical radiotherapy options include stereotactic body radiation therapy (SBRT) and selective internal radiotherapy (SIRT). SBRT delivers ablative doses of radiation in a hypofractionated course and has been used in metastatic disease to the brain, spine, lung, and liver. The local tumor control for SBRT appears to exceed that of conventional fractionation external beam radiotherapy (EBRT). SIRT, also known as radioembolization, is the intraarterial delivery of microspheres impregnated with yttrium-90 (Y90) via the hepatic artery. The evidence is limited to cohort and comparative studies with historical controls; nonetheless, results appear to be promising. Both SBRT and SIRT to liver
\end{abstract}

C.-L. Tsai · J. C.-H. Cheng

Division of Radiation Oncology, Department of Oncology,

National Taiwan University Hospital,

Taipei, Taiwan

\section{J. C.-H. Cheng $(\bowtie)$}

Graduate Institute of Oncology,

National Taiwan University College of Medicine,

Taipei, Taiwan

e-mail: jasoncheng@ntu.edu.tw

H. T. Chung • W. Chu

Department of Radiation Oncology, Odette Cancer Centre, University of Toronto,

Toronto, Canada tumors may serve as a bridge to liver transplantation (LT) and as a treatment strategy for metastasis from colorectal cancer to achieve the effect of metastasectomy. Meanwhile, combining molecular-targeted agents with radiotherapy is an emerging strategy to enhance the therapeutic ratio.

Conclusion SBRT and/or SIRT are promising local ablative modalities for management of unresectable liver tumors. Further well-designed trials are warranted to establish the proper combination of different therapeutic modalities.

Keywords Hepatocellular carcinoma · Liver metastasis . Stereotactic body radiation therapy $\cdot$ Selective internal radiation therapy $\cdot$ Liver transplantation

\section{Introduction}

Hepatic tumors arise either from primary liver cancer (i.e., hepatocellular carcinoma) or metastases to the liver, most commonly from a colorectal primary. The standard curative treatment is surgical resection with tumor-free margins or liver transplantation (LT). Unfortunately, only $30-40 \%$ of patients are resectable [1]. Most hepatic tumors are unresectable at diagnosis due to anatomic location, size, and number of lesions; concurrent nonmalignant liver disease; and significant extrahepatic disease or insufficient hepatic reserve. A number of treatments are in use for unresectable tumors including transarterial chemoembolization (TACE), selective internal radiotherapy (SIRT), stereotactic body radiation therapy (SBRT), external beam radiotherapy (EBRT), systemic and hepatic intraarterial chemotherapy, targeted therapy (e.g., sorafenib), radiofrequency ablation (RFA), microwave ablation, cryotherapy, and other ablative techniques.

Prior to the 1990s, EBRT was reserved primarily in the palliative setting for metastatic disease because of the assumed 
low tolerance of the whole liver to RT. Whole-liver RT achieves a palliative goal in the majority of patients with pain [2]. With the advent of intensity modulated radiotherapy (IMRT) and image-guided radiotherapy (IGRT), higher doses could be delivered safely for partial liver RT. SBRT represents the pinnacle of such technology and permits unprecedented doses of radiation to be delivered with high precision while minimizing radiation exposure to nearby critical structures. Its application is continuously expanding and is currently being used for oligometastases involving the brain, spine, lung, and liver. It is also being used in the management of HCC, mostly as an alternative treatment and in some circumstances as a bridging therapy [3].

SIRT or radioembolization delivers yttrium-90 (Y-90)labeled microspheres into the arteries that supply the tumor. Y-90 microspheres have several advantages as a potential therapy for liver lesions, including the selective delivery of radiolabeled microspheres via the hepatic artery directly into the tumor, as well as limiting the dose to normal liver parenchyma.

The liver is a common site for metastasis from colorectal cancer because of direct venous drainage from the intestines to the liver. Resection with a negative margin in a single operative procedure is the gold standard for limited liver metastases and offers potential for long-term survival. However, most patients are not amenable to potentially curative resection. SBRT and SIRT have arisen as potential alternative treatment choices $[4,5]$.

Orthotopic liver transplantation (OLT) in HCC patients is limited, owing to cancer progression and/or death due to underlying liver disease, while the patient is on the waiting list $[6,7]$. Bridging therapy is thus needed to halt tumor progression and minimize wait list dropout $[8$, 9]. Some patients with advanced HCC could benefit from OLT after downstaging the tumor with neoadjuvant treatment $[10,11]$.

Molecular-targeted therapy surged as a novel treatment choice for advanced HCC. The randomized controlled trial using target therapy, sorafenib, showed median survival and time to radiologic progression were nearly 3 months longer [12]. This review provides an overview of the novel treatments for primary and metastatic tumors of the liver.

\section{External beam radiotherapy}

Historically, the role of EBRT was limited for the liver due to the belief that the normal liver was sensitive to radiation. The dose limitation of whole-liver EBRT is approximately 30 to 35 Gy in 3 to 4 weeks which restricts EBRT to a palliative role [13-17]. In 1987, the Radiation Therapy Oncology Group (RTOG) reported the results of a randomized trial with whole-liver EBRT alone to a dose of $21 \mathrm{~Gy}$ in seven fractions or in combination with the radiosensitizer misonidazole [18]. Although the addition of misonidazole did not significantly improve outcomes, whole-liver EBRT provided a significant palliative benefit, with a decrease in abdominal pain, decreased analgesic requirement in $77 \%$ of patients, less abdominal distention in $67 \%$ of patients, as well as less nausea, vomiting, and anorexia in $40 \%$ of patients.

The subsequent advent of three-dimensional RT treatment planning has expanded the role of EBRT in patients with unresectable liver tumors. Mohiuddin et al. reported an improved median survival if a partial liver boost dose was delivered in conjunction with whole-liver RT for patients with colorectal metastasis to the liver [19]. Later, alternative fractionation schemes were introduced in treating liver tumors. The rapid doubling time of HCC, estimated at 41 days [20], suggests that shortening overall treatment time by hyperfractionation RT may improve response rates. Indeed, twice daily hyperfractionation was used in the University of Michigan series [21-24]. Total dose was individualized based on the volume of normal liver that could be spared without exceeding an estimated $5 \%$ to $10 \%$ risk of radiation-induced liver disease (RILD), considered to be a dose-limiting toxicity. The total dose was the most important factor of survival, with 75 Gy (1.5-Gy fractions twice a day) or more associated with a significantly higher survival (23.9 months) compared to lower doses (14.9 months).

\section{Stereotactic body radiotherapy}

With technical advances in tumor localization and motion management, hypofractionated radiotherapy using imageguided RT is being increasingly adopted as a means to ablate liver tumors, whether primary or metastatic. Focal, highdose SBRT delivers ablative doses in fewer fractions and highly conformal RT volumes. Doses to nearby critical structures and organs are minimized by using very tight margins. Therefore, a robust immobilization device is crucial to ensure reproducible and accurate setup. Respirationinduced tumor motion in the liver is significant and must be controlled during simulation and treatment delivery [25-27]. By doing so, the margin expansion from the gross tumor volume to the planning target volume (PTV) may be reduced. Methods to manage respiratory motion include passive abdominal compression, breath hold, breath coaching, respiratory gating, and respiratory motion tracking $[3$, 28]. Pretreatment verification of tumor position is needed before each session of SBRT to ascertain precise and accurate delivery of SBRT. Image guidance can be accomplished using a megavoltage/kilovoltage cone beam CT or stereoscopic X-rays. 


\section{SBRT for primary liver tumor}

Table 1 summarizes the results of SBRT for primary liver tumor. Choi et al. retrospectively analyzed the outcomes of SBRT for $20 \mathrm{HCC}$ patients, 15 with Child-Pugh A and five with Child-Pugh B disease [29]. The dose was 50 Gy in five or 10 fractions. At a median follow-up of 23 months, the overall response rate was $80 \%$ and 1- and 2-year overall survival rates were $70 \%$ and $43.1 \%$, respectively. The median survival was 20 months. No grade 3 or higher toxicities were noted. In a phase I trial of SBRT (2454 Gy in six fractions) in 31 patients with primary liver tumor, Tse et al. reported a median survival of 13.4 months, 1 -year overall survival of $51 \%$, and 1-year local control of $65 \%$ [30]. No RILD or treatment-related grade $4 / 5$ toxicity was seen within 3 months of SBRT. Grade 3 elevation of liver enzymes was seen in five patients (12\%). Two patients (5\%) with intrahepatic cholangiocarcinoma (IHC) developed transient biliary obstruction, while seven (five HCC and two IHC) had a decline in liver function from Child-Pugh A to B within 3 months after SBRT.

In a phase I dose escalation trial, Cardenes et al. conducted the feasibility of SBRT for HCC in 17 patients with 25 lesions [31]. The dose was escalated from 36 Gy to $48 \mathrm{~Gy}$ in three fractions (one to two fractions per week). No dose-limiting toxicities were observed in the Child-Pugh A patients. Two patients with Child-Pugh B disease developed grade 3 hepatic toxicity at the $42 \mathrm{~Gy}$ (in three fractions) level. The protocol was changed for five subsequent patients with Child-Pugh B to 40 Gy in five fractions, of which one patient experienced progressive liver failure. The only factor related to grade 3 or higher toxicity or death within 6 months after treatment was a Child-Pugh score greater than 8 .

To determine the safety of SBRT for large HCC lesions $(>10 \mathrm{~cm})$, Shin et al. evaluated the outcomes of six patients using regimen of 32-40 Gy in four fractions [32]. Overall, treatment was well tolerated and one patient with a partial response underwent lobectomy after SBRT. Due to advanced disease, 1-year overall survival was only $33 \%$.

\section{SBRT for liver metastases}

Table 2 summarizes the published results of clinical trials of SBRT for liver tumors. From Princess Margaret Hospital, Lee et al. reported on a phase I risk-adapted dose escalation study of SBRT for liver metastases [33]. Individualized radiation doses over six fractions were selected to maintain the same nominal risk of RILD for three estimated risk levels $(5 \%, 10 \%$, and $20 \%)$. The median SBRT dose was $41.8 \mathrm{~Gy}$ in six fractions, with the maximum dose of $60 \mathrm{~Gy}$. The 1 -year local control rate was $71 \%$, and the 18 -month overall survival rate was $47 \%$. Improved local control with

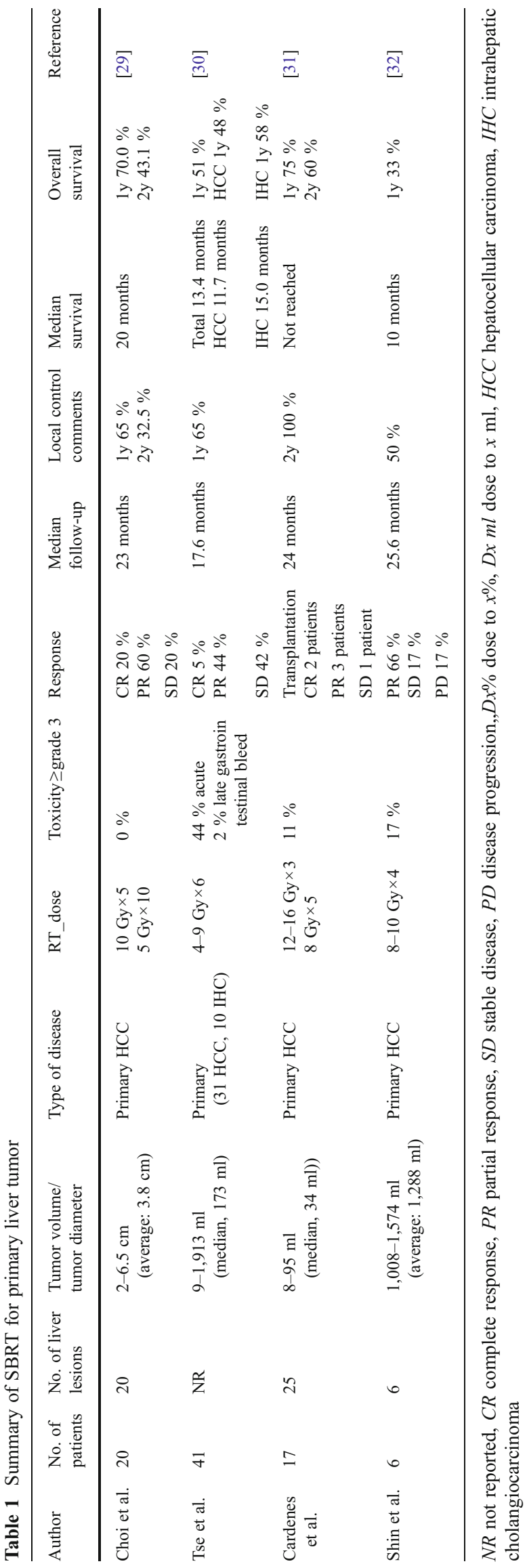




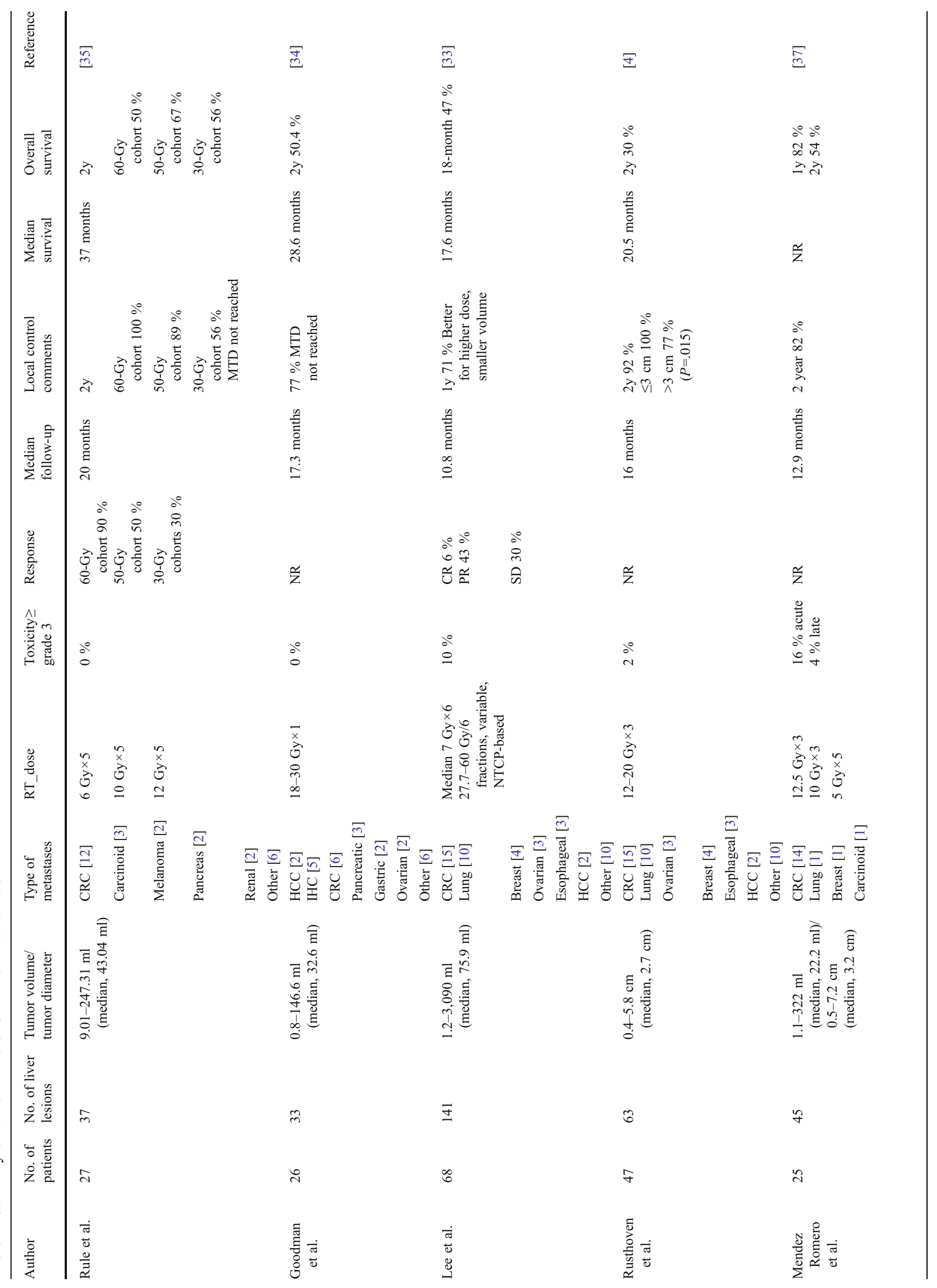


higher doses was found in a univariate analysis. There were only two grade 3 liver enzyme changes but no RILD.

In another phase I dose-escalation study, Goodman et al. reported on the outcomes of 18-30 Gy in a single-fraction SBRT for primary (five patients with IHC, two patients with HCC) or metastatic liver tumors (19 patients) in 26 patients with Child-Pugh A disease [34]. No patients developed dose-limiting toxicities. Across the entire study cohort, the 1 -year local control rate was $77 \%$ and the 2-year actuarial overall survival was $50 \%$. The 1 - and 2-year overall survival rates in patients treated for primary liver tumors were $71 \%$ and $53 \%$, and in those treated for metastatic disease, $61 \%$ and $49 \%$, respectively.

In a phase I dose-escalation study, Rule et al. compared outcomes of $30 \mathrm{~Gy}$ in three fractions, 50 Gy in five fractions, and $60 \mathrm{~Gy}$ in five fractions [35]. Dose-dependent effects on local control of $100 \%, 89 \%$, and $56 \%$ for SBRT doses of $60 \mathrm{~Gy}, 50 \mathrm{~Gy}$, and $30 \mathrm{~Gy}$, respectively, were observed.

Herfarth et al. assessed single-fraction SBRT from 14 to 26 Gy at University of Heidelberg [17, 36]. Thirty-seven patients with 60 tumors were treated, with no toxicity greater than grade 2 observed. The 18 -month local control rate was $68 \%$ for all patients and $81 \%$ for patients who received 20-26 Gy, suggesting a dose response effect. Mendez Romero et al. reported the outcomes of SBRT in 25 patients with primary or metastatic liver lesions. In patients with metastases, $\mathrm{HCC}$ without cirrhosis, and $\mathrm{HCC}<4 \mathrm{~cm}$, the SBRT dose was mostly 37.5 Gy in three fractions [37]. In patients with $\mathrm{HCC} \geq 4 \mathrm{~cm}$ and cirrhosis, the dose was $25 \mathrm{~Gy}$ in five fractions or $30 \mathrm{~Gy}$ in three fractions. The 1- and 2year local tumor control rate for the entire cohort was $94 \%$ and $82 \%$, respectively. The actuarial 1 - and 2-year local control rates were $100 \%$ and $86 \%$ in the metastasis group, respectively. In the HCC group the 1-year local control rate were $75 \%$.

In a compelling multi-institutional dose-escalation phase I/II trial of SBRT for liver metastases, Rusthoven et al. reported on the outcomes of SBRT in 47 patients [4]. The 2-year local control rate was $92 \%$ for all lesions, $100 \%$ (lesions $\leq 3 \mathrm{~cm}$ ) and $77 \%$ (lesions $>3 \mathrm{~cm}$ ). The radiation dose was escalated from 36 to $60 \mathrm{~Gy}$ in three fractions and no dose-limiting toxicity was observed. Grade 3 or greater toxicity was observed only in one patient.

Høyer et al. reported on the outcomes of SBRT for patients with liver metastasis from colorectal carcinoma using a dose of $45 \mathrm{~Gy}$ in three fractions [38]. Median follow-up was 4.3 years. The 2-year actuarial local control rate was $79 \%$. In tumor- and patient-based analyses $(n=141$ total lesions; $n=64$ patients, respectively), the 2 -year local controls were $86 \%$ and $63 \%$, respectively. Nineteen percent had no local or distant progression after 2 years, and 2- and 5 -year overall survival rates were 38 and $13 \%$, respectively. 
In terms of toxicities, one patient died of hepatic failure, one patient underwent surgery for a colonic perforation, and two patients were conservatively treated for duodenal ulcerations [38].

In summary, survival outcomes are better in patients with liver metastasis than HCC. In both groups, there appears to be a dose response for local control. For HCC, the dose of SBRT should be based on the cirrhotic status. For patients with Child-Pugh A cirrhosis, 48 Gy or higher in three fractions is recommended. For patients with Child-Pugh B cirrhosis, more fractionated schemes are recommended (e.g., 40 Gy in five fractions). For liver metastasis, doses greater than $48 \mathrm{~Gy}$ in three fractions or 14-26 Gy in one fraction is recommended. Grade 3 or higher toxicities were more common (up to $44 \%$ ) in patients with HCC and ChildPugh class B cirrhosis. In patients with liver metastasis, no treatment-related grade $4 / 5$ toxicity after SBRT was observed, with grade 3-elevated liver enzymes in less than $20 \%$.

\section{SIRT}

SIRT or radioembolization refers to the delivery of microspheres loaded with radioactive isotopes into the arteries feeding liver tumors. The microsphere is an implantable medical device consisting of resin-based or glass-based biocompatible microspheres loaded with Y-90 [39]. Y-90 is a beta emitter with a half-life of 2.6 days and an average penetration depth in tissues of $2.4 \mathrm{~mm}$. SIRT demonstrated an $89 \%$ treatment response with resin microspheres and $78 \%$ with glass microspheres, respectively, in patients with HCC [40]. In a large retrospective review, Kennedy et al. reported 515 patients receiving 680 treatments of SIRT [41]. Stable disease was seen in $76.8 \%$, partial response in $9.5 \%$, complete response in $4.5 \%$, and progressive liver disease in $9 \%$. The treatment was well tolerated with an incidence of RILD of only $4 \%$ and nonliver grade 3 or greater toxicity of $6 \%$. No relationship was identified in terms of response rates or toxicity by tumor type. Table 3 summarizes the results using SIRT for liver tumors.

\section{SIRT in HCC}

Sangro et al. reported 24 patients treated with resin-based Y90 microspheres, with the delivered activity of $106.3 \mathrm{~Gy}$ [42].The median survival was 7 months, and the time to progression (TTP) was 3 months. In another study by Hilgard et al., HCC patients treated with Y-90 were assessed [43]. Although the median TTP for all patients was 10.0 months, the TTP ranged from 8.0 months for patients with portal vein thrombosis (PVT) to 11.8 months for those without

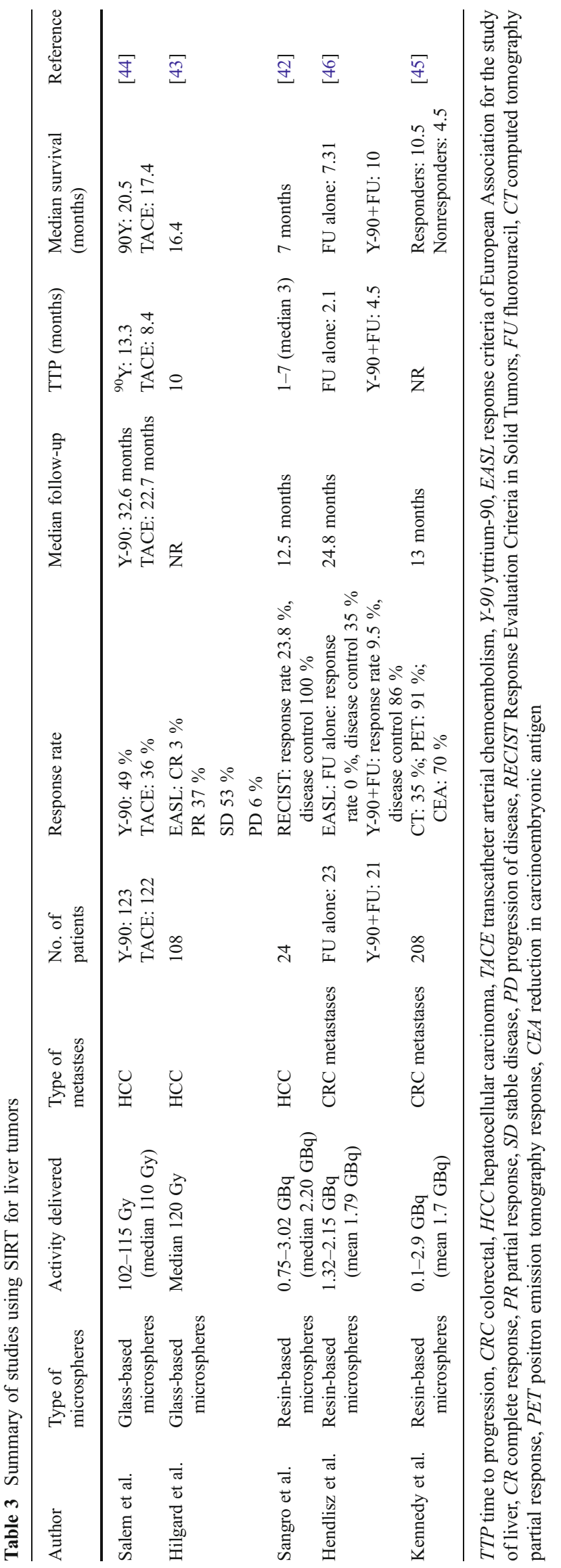


PVT. The 6-month survival probability in the PVT group was $65 \%$ and the median survival was 10.0 months, as compared to $76 \%$ and 16.4 months in patients without detectable PVT.

Recently, Salem et al. analyzed the outcomes comparing Y-90 with transcatheter arterial chemoembolism (TACE) for HCC [44]. There was a trend that patients treated with Y-90 had a higher response rate than with TACE (49\% vs. $36 \%$, $P=.104)$. Although TTP was longer following Y-90 than TACE (13.3 months vs. 8.4 months, $P=.046)$, the median survivals were not statistically different (17.4 months vs. 20.5 months, $P=.232$ ). Among patients with intermediatestage disease, survival was similar between groups of TACE (17.5 months) and Y-90 (17.2 months, $P=.42)$. They concluded that Y-90 leads to longer TTP and less toxicity than TACE, with no survival difference.

\section{SIRT in liver metastases}

SIRT has been used to treat patients with liver metastases. Kennedy et al. assessed Y-90 as a salvage therapy for unresectable colorectal liver metastases [45]. A total of 208 heavily pretreated patients underwent Y-90 delivered through hepatic arterial vessels. Encouraging tumor response was observed on computed tomography, positron emission tomography, and the reduction in carcinoembryonic antigen levels of $35 \%, 91 \%$, and $70 \%$, respectively. The maximum response occurred at approximately 3 months after treatment. Median survival was 10.5 months in the responders but only 4.5 months in the nonresponders.

Hendlisz et al. compared intravenous fluorouracil alone (FU) with the combination of Y-90 and intravenous fluorouracil (Y-90+FU) [46]. The median survival were 7.3 and 10.0 months in FU and Y-90+FU, respectively $(P=.80)$. The median TTP was 2.1 and 4.5 months, respectively $(P=03)$. Grade 3 or 4 toxicities were recorded in six patients after FU and in one after $\mathrm{Y}-90+\mathrm{FU}(P=.10)$. They concluded that combination therapy is a safe and effective therapeutic option for chemotherapy-refractory liver metastases.

In summary, Y-90 is another promising treatment for patients with multifocal liver tumors that have failed from previous treatments. Response rates range from $24 \%$ to $49 \%$ in $\mathrm{HCC}$ and $9.5 \%$ to $91 \%$ in liver metastasis. Treatment-related complications, such as ulceration of stomach or duodenum and RILD, were few and manageable. No patient experienced RILD after SIRT.

\section{SIRT and SBRT as a bridge to LT}

LT is an effective treatment for HCC other than surgical resection. LT has the advantages of a resected malignancy and elimination of parenchymal liver disease that initially led to HCC. A landmark study from the Milan group reported survival at 4 years of $85 \%$, with a recurrence rate of $8 \%$ in an appropriately selected patient group [47]. The Milan criteria suggested in this study remain the benchmark for providing a priority status for HCC patients who are otherwise candidates for LT [48]. The major criteria include a single tumor $\leq 5 \mathrm{~cm}$, up to three nodules $\leq 3 \mathrm{~cm}$ each, and no macrovascular invasion.

However, some patients initially meeting the Milan criteria may not make it to LT because of tumor progression. There are also a significant number of patients with advanced stage of disease at diagnosis who were not ideal candidates for LT. Therefore, several bridging strategies have been developed, including RFA, percutaneous ethanol injection (PEI), and TACE [49-52]. In the first prospective study on downstaging of HCC prior to LT, a combination of TACE, RFA, PEI, and/or surgical resection was investigated [53]. In this carefully selected patient group, $70 \%$ were successfully downstaged, and $53 \%$ eventually had a LT. At a median follow-up of 16 months, no HCC recurrence was observed.

The role of SIRT with Y-90 for downstaging HCC has also been investigated [7, 54]. For T3 unresectable HCC, $56 \%$ were downstaged from T3 to T2, while $23 \%$ were downstaged and underwent LT following treatment. Comparing the downstaging efficacy of TACE and SIRT with Y-90, the partial response rate was more favorable by SIRT and Y-90 than TACE (61 \% vs. $37 \%$ ). HCC downstaged to T2 was achieved in $31 \%$ by TACE and $58 \%$ by SIRT and Y-90.

SBRT is now emerging as another treatment option for bridging therapy [10, 55-57]. Table 4 summarizes the results of SBRT as a bridge for LT.

Al Hamad et al. published the first report of a $\mathrm{HCC}$ patient undergoing SBRT with $50 \mathrm{~Gy}$ in five fractions while awaiting LT. Following LT, pathological examination of the liver explant revealed only scarring at the irradiated site [58].

Sandroussi et al. reported their experience in 10 patients with either failure from prior local therapies or unsuitability for standard local therapies [56]. The safety and efficacy of SBRT as a bridging therapy was evaluated. The median SBRT delivered dose was 33 Gy (range 8.5-54 Gy) in one to six fractions. Nine patients completed their SBRT as planned and one patient was transplanted after the first fraction. Two tumors remained stable, and the others had $10-50 \%$ regression, sustained on follow-up images. Two patients were delisted as a result of cancer progression outside the treated field. Three patients were still waiting for LT. Five patients who underwent LT had a demonstrable pathological response with the degree of necrosis ranging from $40 \%$ to $90 \%$. With a median follow-up of 14 months, all five patients survived with no disease progression. 


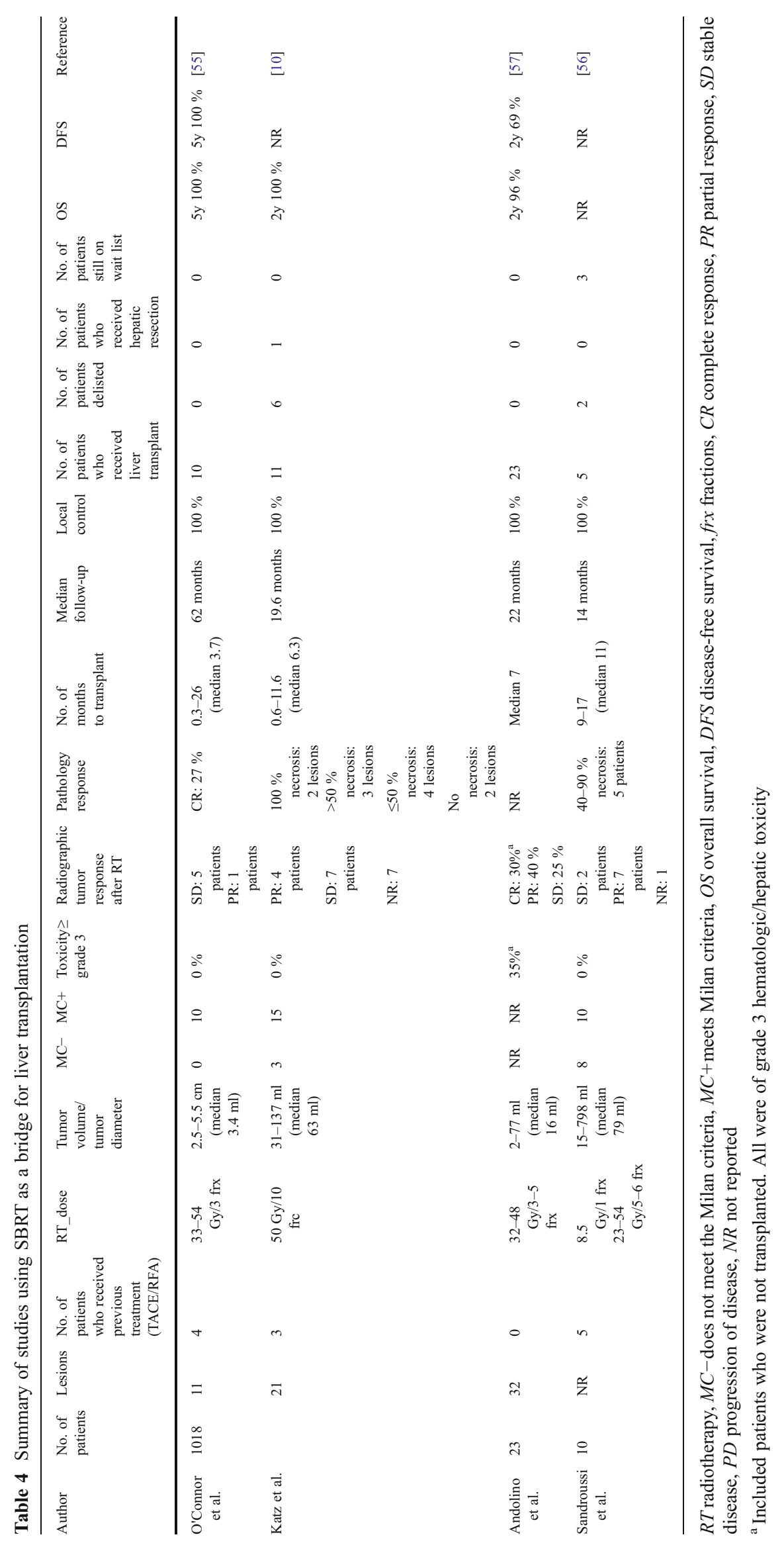


The largest series, reported by Andolino et al., described 23 of 60 patients with liver-confined HCC undergoing LT after SBRT [57]. The median dose was $44 \mathrm{~Gy}$ in three fractions for those with Child-Pugh A cirrhosis and 40 Gy in five fractions for those with Child-Pugh B cirrhosis. The median time to transplant was 7 months. Neither median progression-free survival nor overall survival has yet to be achieved at the time of the report. The actuarial rates at 2 years were $69 \%$ and $96 \%$, respectively. For the entire cohort, the rates of complete response, partial response, and stable disease were $30 \%, 40 \%$, and $25 \%$, respectively. Of the 21 patients ( $35 \%$ ) who developed grade 3 hematologic/ hepatic toxicity, 17 had preexisting grade 2 dysfunction. Patients who did not undergo transplant were 2.7 times more likely to experience progression and 17.1 times more likely to die than were patients who did undergo transplant.

Another study by Katz et al. retrospectively reviewed 18 patients receiving SBRT with a median dose of $50 \mathrm{~Gy}$ in 10 fractions [10]. Six of 18 patients were delisted because of disease progression or other causes. Twelve patients successfully underwent major hepatic resection (one patient) or LT (11 patients). Ten patients with 11 lesions were evaluable for pathological response, and five of them had more than $50 \%$ necrosis. All patients were alive after LT and/or hepatic resection at a median follow-up of 19.6 months. Only one patient developed intrahepatic recurrence.

$\mathrm{O}^{\prime}$ Connor et al. reported a series of $10 \mathrm{HCC}$ patients undergoing SBRT as a bridge to LT [55]. The median dose was $51 \mathrm{~Gy}$ in three fractions. Median time from SBRT to LT was 62 months. Based on explant pathology, $27 \%$ of patients had complete tumor necrosis. All the remaining patients' tumors decreased or remained stable in size. The median survival was not reached. The 5 -year overall and disease-free survival were $100 \%$.

In summary, SIRT and SBRT may be practically considered as a bridge to LT while awaiting a donor. Some patients may benefit from SIRT (23-53\%) or SBRT (50-100\%) with the downstaging effect to meet the LT criteria [7, 53]. The results of SIRT and SBRT are complimentary to each other as an adjunct to LT.

\section{Future directions}

Sorafenib (Nexavar; Bayer, West Haven, CT, and Onyx, Richmond, CA) is a molecular-targeted therapy used in the palliative treatment of advanced HCC in Child-Pugh A or selected patients with Child-Pugh B. Its clinical use is supported by the results of two randomized phase III trials (the SHARP trial and the Asia Pacific trial). In the SHARP trial, the median TTP was 5.5 months and 2.8 months with sorafenib and placebo, respectively [12]. The 1-year survival rates were $44 \%$ and $33 \%$ with sorafenib and placebo, respectively. Among patients with hepatitis $\mathrm{C}$, median overall survival (14.0 vs. 7.9 months) and median TTP (7.6 vs. 2.8 months) were significantly prolonged with sorafenib. In the Asia Pacific trial, the median TTP were 2.8 months and 1.4 months with sorafenib and placebo, respectively [59]. The 6-month survival rates were $53 \%$ and $37 \%$ with sorafenib and placebo, respectively. Of note is that no patient in both trials achieved a complete response.

Because of the modest benefits by sorafenib, the combination of sorafenib and RT is being studied. In a case report, combined sorafenib and RT resulted in marked tumor shrinkage [60]. In an early experience of 20 HCC patients receiving the combined regimen, $22 \%$ in-field response was observed. Other potential radiosensitizers are under investigation, such as sunitinib (Sutent; Pfizer, New York, NY) and AR-42 (Arno Therapeutics, Inc., Fairfield, NJ). [61, 62]. To date, there is no randomized trial evaluating the combination of a molecular-targeted radiosensitizer with RT in the treatment of HCC.

\section{Conclusion}

SBRT and/or SIRT are promising local ablative modalities for patients with unresectable liver tumors. Currently, supportive data is limited to phase 1 or 2 studies. Future research will focus on combining different therapeutic modalities, including SBRT, SIRT, TACE, and novel targeting agents.

Conflict of interest The authors declare that they have no conflicts of interest.

\section{References}

1. Bruix J, Llovet JM (2002) Prognostic prediction and treatment strategy in hepatocellular carcinoma. Hepatology 35:519-524

2. Borgelt BB, Gelber R, Brady LW et al (1981) The palliation of hepatic metastases: results of the Radiation Therapy Oncology Group pilot study. Int J Radiat Oncol Biol Phys 7:587-591

3. Lo SS, Fakiris AJ, Chang EL et al (2010) Stereotactic body radiation therapy: a novel treatment modality. Nat Rev Clin Oncol 7:44-54

4. Rusthoven KE, Kavanagh BD, Cardenes H et al (2009) Multiinstitutional phase I/II trial of stereotactic body radiation therapy for liver metastases. J Clin Oncol 27:1572-1578

5. Memon K, Lewandowski RJ, Kulik L et al (2011) Radioembolization for primary and metastatic liver cancer. Semin Radiat Oncol 21:294-302

6. Llovet JM, Fuster J, Bruix J (1999) Intention-to-treat analysis of surgical treatment for early hepatocellular carcinoma: resection versus transplantation. Hepatology 30:1434-1440

7. Kulik LM, Atassi B, van Holsbeeck L et al (2006) Yttrium-90 microspheres (TheraSphere) treatment of unresectable 
hepatocellular carcinoma: downstaging to resection, RFA and bridge to transplantation. J Surg Oncol 94:572-586

8. Pelletier SJ, Fu S, Thyagarajan V et al (2009) An intention-to-treat analysis of liver transplantation for hepatocellular carcinoma using organ procurement transplant network data. Liver Transpl 15:859868

9. Maggs JR, Suddle AR, Aluvihare V et al (2012) Systematic review: the role of liver transplantation in the management of hepatocellular carcinoma. Aliment Pharmacol Ther.

10. Katz AW, Chawla S, Qu Z, et al (2011) Stereotactic hypofractionated radiation therapy as a bridge to transplantation for hepatocellular carcinoma: clinical outcome and pathologic correlation. Int J Radiat Oncol Biol Phys.

11. Khalaf H, Alsuhaibani H, Al-Sugair A et al (2010) Use of yttrium90 microsphere radioembolization of hepatocellular carcinoma as downstaging and bridge before liver transplantation: a case report. Transplant Proc 42:994-998

12. Llovet JM, Ricci S, Mazzaferro V et al (2008) Sorafenib in advanced hepatocellular carcinoma. N Engl J Med 359:378-390

13. Lawrence TS, Ten Haken RK, Kessler ML et al (1992) The use of 3-D dose volume analysis to predict radiation hepatitis. Int J Radiat Oncol Biol Phys 23:781-788

14. Jackson A, Ten Haken RK, Robertson JM et al (1995) Analysis of clinical complication data for radiation hepatitis using a parallel architecture model. Int J Radiat Oncol Biol Phys 31:883-891

15. Burman C, Kutcher GJ, Emami B et al (1991) Fitting of normal tissue tolerance data to an analytic function. Int $\mathrm{J}$ Radiat Oncol Biol Phys 21:123-135

16. Emami B, Lyman J, Brown A et al (1991) Tolerance of normal tissue to therapeutic irradiation. Int J Radiat Oncol Biol Phys 21:109-122

17. Herfarth KK, Debus J, Lohr F et al (2001) Stereotactic single-dose radiation therapy of liver tumors: results of a phase I/II trial. J Clin Oncol 19:164-170

18. Leibel SA, Pajak TF, Massullo V et al (1987) A comparison of misonidazole sensitized radiation therapy to radiation therapy alone for the palliation of hepatic metastases: results of a Radiation Therapy Oncology Group randomized prospective trial. Int J Radiat Oncol Biol Phys 13:1057-1064

19. Mohiuddin M, Chen E, Ahmad N (1996) Combined liver radiation and chemotherapy for palliation of hepatic metastases from colorectal cancer. J Clin Oncol 14:722-728

20. Johnson PJ, Williams R (1980) Serum alpha-fetoprotein estimations and doubling time in hepatocellular carcinoma: influence of therapy and possible value in early detection. J Natl Cancer Inst 64:1329-1332

21. Robertson JM, Lawrence TS, Andrews JC et al (1997) Long-term results of hepatic artery fluorodeoxyuridine and conformal radiation therapy for primary hepatobiliary cancers. Int J Radiat Oncol Biol Phys 37:325-330

22. Lawrence TS, Kessler ML, Robertson JM (1993) Conformal highdose radiation plus intraarterial floxuridine for hepatic cancer. Oncology (Williston Park) 7:51-57, discussion 57-58, 63

23. Dawson LA, McGinn CJ, Normolle D et al (2000) Escalated focal liver radiation and concurrent hepatic artery fluorodeoxyuridine for unresectable intrahepatic malignancies. J Clin Oncol 18:22102218

24. Ben-Josef E, Normolle D, Ensminger WD et al (2005) Phase II trial of high-dose conformal radiation therapy with concurrent hepatic artery floxuridine for unresectable intrahepatic malignancies. J Clin Oncol 23:8739-8747

25. Lo SS, Cardenes HR, Teh BS et al (2008) Stereotactic body radiation therapy for nonpulmonary primary tumors. Expert Rev Anticancer Ther 8:1939-1951

26. Dawson LA, Eccles C, Bissonnette JP et al (2005) Accuracy of daily image guidance for hypofractionated liver radiotherapy with active breathing control. Int J Radiat Oncol Biol Phys 62:12471252

27. Kirilova A, Lockwood G, Choi P et al (2008) Three-dimensional motion of liver tumors using cine-magnetic resonance imaging. Int J Radiat Oncol Biol Phys 71:1189-1195

28. Chang BK, Timmerman RD (2007) Stereotactic body radiation therapy: a comprehensive review. Am J Clin Oncol 30:637-644

29. Choi BO, Jang HS, Kang KM et al (2006) Fractionated stereotactic radiotherapy in patients with primary hepatocellular carcinoma. Jpn J Clin Oncol 36:154-158

30. Tse RV, Hawkins M, Lockwood G et al (2008) Phase I study of individualized stereotactic body radiotherapy for hepatocellular carcinoma and intrahepatic cholangiocarcinoma. J Clin Oncol 26:657-664

31. Cárdenes H, Price T, Perkins S et al (2010) Phase I feasibility trial of stereotactic body radiation therapy for primary hepatocellular carcinoma. Clinical and Translational Oncology 12:218-225

32. Shin YJ, Kim MS, Yoo SY et al (2010) Pilot study of stereotactic body radiotherapy for huge hepatocellular carcinoma unsuitable for other therapies. Tumori 96:65-70

33. Lee MT, Kim JJ, Dinniwell R et al (2009) Phase I study of individualized stereotactic body radiotherapy of liver metastases. J Clin Oncol 27:1585-1591

34. Goodman KA, Wiegner EA, Maturen KE et al (2010) Doseescalation study of single-fraction stereotactic body radiotherapy for liver malignancies. Int J Radiat Oncol Biol Phys 78:486-493

35. Rule W, Timmerman R, Tong L et al (2011) Phase I doseescalation study of stereotactic body radiotherapy in patients with hepatic metastases. Ann Surg Oncol 18:1081-1087

36. Herfarth KK, Debus J, Wannenmacher M (2004) Stereotactic radiation therapy of liver metastases: update of the initial phaseI/II trial. Front Radiat Ther Oncol 38:100-105

37. Mendez Romero A, Wunderink W, Hussain SM (2006) Stereotactic body radiation therapy for primary and metastatic liver tumors: a single institution phase I-II study. Acta Oncol 45:831-837

38. Hoyer M, Roed H, Traberg Hansen A (2006) Phase II study on stereotactic body radiotherapy of colorectal metastases. Acta Oncol 45:823-830

39. Sarfaraz M, Kennedy AS, Cao ZJ et al (2003) Physical aspects of yttrium-90 microsphere therapy for nonresectable hepatic tumors. Med Phys 30:199-203

40. Vente MA, Wondergem M, van der Tweel I et al (2009) Yttrium-90 microsphere radioembolization for the treatment of liver malignancies: a structured meta-analysis. Eur Radiol 19:951-959

41. Kennedy AS, McNeillie P, Dezarn WA et al (2009) Treatment parameters and outcome in 680 treatments of internal radiation with resin 90Y-microspheres for unresectable hepatic tumors. Int J Radiat Oncol Biol Phys 74:1494-1500

42. Sangro B, Bilbao JI, Boan J et al (2006) Radioembolization using 90Y-resin microspheres for patients with advanced hepatocellular carcinoma. Int J Radiat Oncol Biol Phys 66:792-800

43. Hilgard P, Hamami M, Fouly AE et al (2010) Radioembolization with yttrium-90 glass microspheres in hepatocellular carcinoma: European experience on safety and long-term survival. Hepatology 52:1741-1749

44. Salem R, Lewandowski RJ, Kulik L (2011) Radioembolization results in longer time-to-progression and reduced toxicity compared with chemoembolization in patients with hepatocellular carcinoma. Gastroenterology 140:497-507, e492

45. Kennedy AS, Coldwell D, Nutting C et al (2006) Resin 90Ymicrosphere brachytherapy for unresectable colorectal liver metastases: modern USA experience. Int J Radiat Oncol Biol Phys 65:412-425

46. Hendlisz A, Van den Eynde M, Peeters M et al (2010) Phase III trial comparing protracted intravenous fluorouracil infusion alone or with yttrium-90 resin microspheres radioembolization for liver- 
limited metastatic colorectal cancer refractory to standard chemotherapy. J Clin Oncol 28:3687-3694

47. Mazzaferro V, Regalia E, Doci R et al (1996) Liver transplantation for the treatment of small hepatocellular carcinomas in patients with cirrhosis. N Engl J Med 334:693-699

48. Sharma P, Balan V, Hernandez JL et al (2004) Liver transplantation for hepatocellular carcinoma: the MELD impact. Liver Transpl $10: 36-41$

49. Veltri A, Grosso M, Martina MC et al (1998) Effect of preoperative radiological treatment of hepatocellular carcinoma before liver transplantation: a retrospective study. Cardiovasc Intervent Radiol 21:393-398

50. Maddala YK, Stadheim L, Andrews JC et al (2004) Drop-out rates of patients with hepatocellular cancer listed for liver transplantation: outcome with chemoembolization. Liver Transpl 10:449-455

51. Huo TI, Huang YH, Su CW et al (2008) Validation of the HCCMELD for dropout probability in patients with small hepatocellular carcinoma undergoing locoregional therapy. Clin Transplant 22:469-475

52. Cucchetti A, Cescon M, Bigonzi E et al (2011) Priority of candidates with hepatocellular carcinoma awaiting liver transplantation can be reduced after successful bridge therapy. Liver Transpl 17:1344-1354

53. Yao FY, Hirose R, LaBerge JM et al (2005) A prospective study on downstaging of hepatocellular carcinoma prior to liver transplantation. Liver Transpl 11:1505-1514

54. Lewandowski RJ, Kulik LM, Riaz A et al (2009) A comparative analysis of transarterial downstaging for hepatocellular carcinoma: chemoembolization versus radioembolization. Am J Transplant 9:1920-1928

55. O'Connor JK, Trotter J, Davis GL (2012) et al. Long-term outcomes of stereotactic body radiation therapy in the treatment of hepatocellular cancer as a bridge to transplantation, Liver Transpl

56. Sandroussi C, Dawson LA, Lee M et al (2010) Radiotherapy as a bridge to liver transplantation for hepatocellular carcinoma. Transpl Int 23:299-306

57. Andolino DL, Johnson CS, Maluccio M et al (2011) Stereotactic body radiotherapy for primary hepatocellular carcinoma. Int $\mathrm{J}$ Radiat Oncol Biol Phys 81:e447-453

58. Al Hamad AA, Hassanain M, Michel RP (2009) Stereotactic radiotherapy of the liver: a bridge to transplantation stereotactic radiotherapy of the liver: a bridge to transplantation. Technol Cancer Res Treat 8:401-405

59. Cheng AL, Kang YK, Chen Z et al (2009) Efficacy and safety of sorafenib in patients in the Asia-Pacific region with advanced hepatocellular carcinoma: a phase III randomised, double-blind, placebo-controlled trial. Lancet Oncol 10:25-34

60. Hsieh CH, Jeng KS, Lin CC et al (2009) Combination of sorafenib and intensity modulated radiotherapy for unresectable hepatocellular carcinoma. Clin Drug Investig 29:65-71

61. Lu YS, Chou CH, Tzen KY (2012) et al. Radiosensitizing effect of a phenylbutyrate-derived histone deacetylase inhibitor in hepatocellular carcinoma, Int J Radiat Oncol Biol Phys

62. Chi KH, Liao CS, Chang CC et al (2010) Angiogenic blockade and radiotherapy in hepatocellular carcinoma. Int $\mathrm{J}$ Radiat Oncol Biol Phys 78:188-193 